\title{
Reversible Formation of Gold Halides in Single-Crystal Hybrid-Perovskite/Au Interface upon Biasing and Effect on Electronic Carrier Injection
}

\author{
Jan Pospisil ${ }^{1}$, Antonio Guerrero ${ }^{2 *}$, Oldrich Zmeskal $^{1}$, Martin Weiter ${ }^{1}$, \\ Juan Jesus Gallardo, ${ }^{3}$ Javier Navas, ${ }^{3}$ and Germà Garcia-Belmonte ${ }^{2 *}$ \\ ${ }^{1}$ Brno University of Technology, Faculty of Chemistry, Purkyňova 118, 61200 Brno, \\ Czech Republic \\ ${ }^{2}$ Institute of Advanced Materials (INAM), Universitat Jaume I, 12006 Castelló, Spain \\ ${ }^{3}$ Departamento de Química Física, Facultad de Ciencias, Universidad de Cádiz, E- \\ 11510 Puerto Real (Cádiz), Spain
}

*Email: aguerrer@uji.es, garciag@uji.es

27 August 2019

\begin{abstract}
Solar cells, light emitting diodes, X-rays detectors and other electronic devices based on perovskite materials often incorporate gold electrodes, either in direct or indirect contact with the perovskite compound. Whilst it is widely recognized that the external contacts are an essential part of any electronic device, quite often chemical interactions between active layers and contacts deteriorate the operation and induce degradation, being the identification of the chemical nature of such interfacial structures an open question. This work reveals that chemical reactivity of the gold in contact with the perovskite semiconductor leads to the reversible formation of oxidized gold halide species and explains generation of halide vacancies in the vicinity of the interface. Such a reaction is induced by electrical biasing and produces modifications of the current level by favoring the ability of perovskite/Au interfaces to inject electronic carriers. It is shown that the current injection increment does not depend on the halogen source used, either extrinsic by iodine vapor sublimation of Au electrodes, or intrinsic by bias-driven migration of bromide ions. The current level has mainly an electronic origin, in such a way that the increment/suppression of injected currents is connected to the interface reactivity assisted by the intrinsic halogen ion migration inside the perovskite bulk. In addition, it is confirmed the formation of a dipole-like structures at the reacted electrode lowering the potential barrier for electronic carriers. Our findings confirm the important role of adequate selection of the external contacts and suggest progressing in a deeper understanding of contact reactivity as it dominates the operation characteristics, rather
\end{abstract}


than being governed by bulk transport properties of the charge carriers, either electronic or ionic. 


\section{Introduction}

Perovskite solar cells (PSCs) have shown rapid improvements in efficiencies, above $20 \%$ in the last few years, ${ }^{[1]}$ indicating the promise of this technology for a prominent role in clean energy conversion in the near future. In addition to photovoltaic applications, perovskite-based materials are being explored as sensitive layers for highenergy radiation detectors and imaging devices for medical diagnostics. ${ }^{[2]}$ These detection technologies rely upon the ability of perovskite compounds of absorbing highenergy photons and convert their energy into electronic carriers that are finally collected at the outer contacts. For soft X-ray photons $(<10 \mathrm{keV})$, absorbing layer thickness of tens of $\mu \mathrm{m}$ suffices to stop the radiation. But hard X-ray radiation (10-50 keV) possesses much longer penetration length $(\sim 100 \mu \mathrm{m})$ in compounds as methylammonium lead iodide or bromide $\left(\mathrm{MAPbI}_{3}, \mathrm{MAPbBr}_{3}\right) .{ }^{[3]}$ Hence, relatively thick layers $(0.1-1 \mathrm{~mm})$ need to be employed to achieve sufficient electrical signal. Despite the large electronic carrier mobility and mobility-lifetime product, ${ }^{[4,5]}$ such thick absorbing layers should be externally biased in order to increase the detector responsivity. Electrical fields as high as $\sim 0.1-1 \mathrm{~V} \mu \mathrm{m}^{-1}$ are commonly needed for poly-crystalline perovskite deposits incorporated into X-ray detectors. ${ }^{[3,5]}$ Large field strength requirements are in some amount lowered when devising single-crystal approaches for X-ray detection using perovskite materials. ${ }^{[4,6]}$ In any case, it is widely recognized that the application of an external bias promotes the displacement and interfacial built-up of intrinsic mobile ions. ${ }^{[7,8]}$ Ion accumulation finally shields the electrical field within the absorbing layer bulk, reducing as a consequence the detector sensitivity. The applicability of perovskite compounds for gamma photon $(50 \mathrm{keV}-1 \mathrm{MeV})$ detection faces similar issues caused by electrical biasing. ${ }^{[9-11]}$

Apart from electric field shielding, mobile ions might chemically interact with the materials at the outer electrodes. Migrating halides approaching the contacts, in the presence of high concentrations of electrons and holes, can produce electrochemical reactions. For example, generation of molecular $I_{2}$ has been reported which in turn induces electrochemical reactions like the oxidation of the external metal electrode to generate the corresponding halides, ${ }^{[12,13]}$ i.e. Ag to AgI, where the formal oxidation state of the metal is modified from 0 to +1 as detected several times. Electrochemical reactions have also been observed with the commonly used spiro-OMeTAD hole extraction layer, which can be reduced producing highly resistive neutral species. ${ }^{[14]}$ Alternatively, anion exchange reactions with the anions of the external contacts have also been reported like in the transformation of $\mathrm{ZnO}$ into $\mathrm{ZnI}_{2}{ }^{2}$ Moreover, reversible reactions with $\mathrm{TiO}_{2}$ have also been detected by Raman spectroscopy which are related to hysteresis in the $j$ - $V$ measurements. ${ }^{[15]}$ The implications of this chemical reactivity is very important as it totally modifies the energy levels and injection barriers at the contacts and may induce performance degradation. ${ }^{[14]}$ Apart from degradation caused by chemical interactions between intrinsic mobile ions and device constituents, extrinsic molecules (moisture, oxygen) are known to severely affect performance. Some strategies (encapsulation, interface engineering) to address that issue have been 
reported. ${ }^{[16]}$ Interestingly, post-device ligand treatments have been suggested consisting on direct reaction with ligand vapors to form stable low-dimensional perovskite complexes at the contact surface. ${ }^{[17]}$

As far as high-energy photon detectors is concerned, usual contacting materials include metals of reduced reactivity. For instance, interdigitate $\mathrm{Au} / \mathrm{Ti}$ electrodes were employed to analyze conductive properties of spin- or spray-coated $\mathrm{MAPbI}_{3} \mathrm{X}$-ray sensitive layers. ${ }^{[3]}$ To enhance cathode electron extraction in single-crystal $\mathrm{MAPbBr}_{3}$ detectors, electron-transporting layers comprising $20 \mathrm{~nm} \mathrm{C}_{60}$ and $8 \mathrm{~nm} \mathrm{BCP}$ interlayers were deposited on the bottom surface before Ag metal contact, while an $\mathrm{Au}(25 \mathrm{~nm})$ anode was evaporated on the top surface. ${ }^{[4]}$ More sophisticated contacting materials are formed by poly(3,4-ethylenedioxythiophene) polystyrene sulfonate (PEDOT:PSS) and phenyl- $\mathrm{C}_{61}$-butyric acid methyl ester (PCBM) and $\mathrm{ZnO}$ as hole-selective and electronselective contact and buffer layers, respectively. ${ }^{[5]}$ In any case, gold is still extensively used either in direct contact with the perovskite absorber, ${ }^{[4,}{ }^{18]}$ or covering proper interlayers. ${ }^{[6]}$ However, it is already known that $\mathrm{Au}$ may interact with the sensitive perovskite layers causing degradation. For instance, gold migrates through the holetransporting layer spiro-OMeTAD, and reacts with the perovskite material affecting the device performance metrics under working photovoltaic conditions. ${ }^{[19,20]}$ Therefore, direct interactions of perovskite compounds and, specifically, their mobile ionic constituents with gold is an issue that deserves further analysis because of its important technological implications. This will be then the main subject of this work. Here singlecrystal $\mathrm{MAPbBr}_{3}$ is investigated in order to focus on contact reactivity effects and avoid grain-boundary interferences. Bromide perovskite compound is also chosen because of its greater ambient stability in comparison with iodide counterparts.

It is reported here that electrical biasing produces modifications of the current level by favoring the ability of perovskite/Au interfaces to inject electronic carriers. Current level is determined by formation of new species comprising $\mathrm{Au}-\mathrm{X}$ (Au in oxidation state I or III) bonds ( $\mathrm{X}=\mathrm{I}, \mathrm{Br})$ that appear at the surface of the electrodes. Moreover, the effect of favoring current injection is observed to be highly similar irrespective of the halogen source, either extrinsic by iodine vapor sublimation of $\mathrm{Au}$ electrodes, or intrinsic by bias-driven migration of bromide ions. In addition, it is confirmed the formation of a dipole-like structures at the reacted electrode that lower the carrier potential barrier. Our findings confirm the important role mobile intrinsic defects have on the device operation and performance deterioration produced by contact reactivity.

\section{Results and Discussion}

In this work monocrystalline $\mathrm{MAPbBr}_{3}$ perovskite samples were prepared by using the inverse temperature crystallization method as described by Bakr et al. ${ }^{[21]}$, and conditions are described in the methods section. Symmetric devices are fabricated by evaporation of gold contacts in opposite sides of the single crystals, see inset of 
Figure 1, and encapsulated to avoid moisture penetration. Measurements of $j-V$ curves were carried out in the dark at room temperature for different fresh crystals that have not been previously electrically biased. Current flowing through the device was measured initially from $0 \mathrm{~V}$ towards either positive or negative applied bias as in Figure $1 \mathrm{a}$ and $1 \mathrm{~b}$, respectively. Symmetrical contacts would entail symmetric $j-V$ curves. However, preferential direction in one quadrant is observed when sweeping the voltage at low scan rates of $50 \mathrm{mV} \mathrm{s}^{-1}$, indicating that biasing distorts the symmetric response. Interestingly, the initial bias direction determines the quadrant at which the current level is preferentially favored. Indeed, if the device is initially polarized towards positive voltages, high current is observed at positive voltages (Figure 1a) and the opposite is observed when the device is initially polarized towards negative values (Figure 1b). Next, in order to understand if the injection of carriers can be further promoted by poling the device at a fixed voltage, the two devices were polarized either at $+5 \mathrm{~V}$ (Figure 1a) or $-5 \mathrm{~V}$ (Figure 1b) during $120 \mathrm{~s}$. As can be observed in Figure 1, this polarization approximately doubles the injection current. Alternatively, the current can be partially or totally suppressed if polarization is carried out at the opposite quadrant, where current injection is not favored. Here, it is important to note that magnitudes of current and degree in which polarization is suppressed varies from sample to sample, but we have recurrently observed that the overall effect is largely reproducible. It is also noticeable that in the direction of preferential injection, current hysteresis is further promoted.

The previously reported experiment leads us to consider outer interfacial effects on the device current driven by the applied bias. In particular, here we propose that reversible reactions at the contact layer modify the interface electronic properties after halogen interaction with the $\mathrm{Au}$ electrode. As later demonstrated, the observed current has preferentially an electronic origin, in such a way that the increment/suppression of injected currents is connected to the interface reactivity assisted by the intrinsic halogen ion migration inside the perovskite bulk.

One related observation is the often observed generation of a remnant open-circuit potential $\left(V_{\text {oc }}\right)$ in the device after polarization that persists for long time. ${ }^{[22]}$ In particular, a device that has not been previously polarized shows a $V_{\mathrm{oc}}=0 \mathrm{~V}$ as expected for a symmetric device. However, after poling during $5 \mathrm{~min}$ it typically shows a $V_{\text {oc }}=0.5-0.6$ $\mathrm{V}$, pointing to the presence of asymmetric contacts with some electrostatic charges remaining in one of them. This suggests us the formation of a dipole-like structure at the reacted electrode ${ }^{[8]}$ with surface charge concentration of order $10^{14} \mathrm{~cm}^{-2}$ resulting from simple electrostatic calculations. The net electrical effect is then lowering the potential barrier for electronic carriers at interfaces. The open question is now identifying the chemical nature of such interfacial structures. 


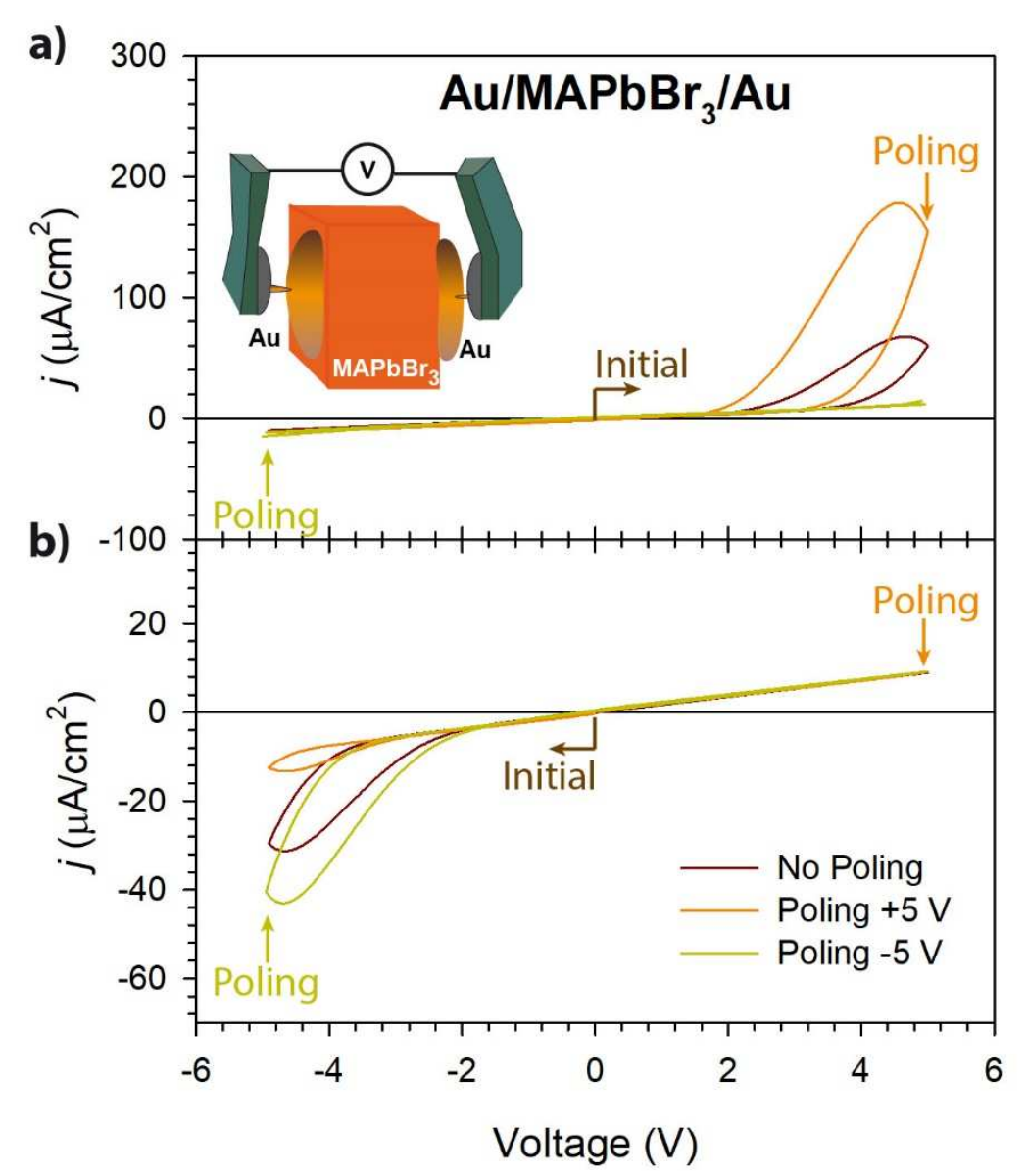

Figure 1: $j$ - $V$ curves measured at scan rate of $50 \mathrm{mV} / \mathrm{s}$ of symmetric singlecrystal-based $\mathrm{Au} / \mathrm{MAPbBr}_{3} / \mathrm{Au}$ devices, initially from $0 \mathrm{~V}$ towards either positive voltages (a) or negative applied bias (b). Devices are next poled at the quadrant where injection is observed $+5 \mathrm{~V}$ (a) and $-5 \mathrm{~V}$ (b). Finally, devices are poled in the opposite bias and injection current is mostly suppressed. The inset shows the device configuration.

We next investigate the effect of the scan rate on the electrical response of symmetrical device structure. However, it is adopted here a sandwich configuration (Figure 2a) since it provides superior experimental versatility as later explained. Interestingly, if a fresh and previously non-polarized device is first measured at high scan rates of $2000 \mathrm{mV} \mathrm{s}^{-1}$ (Figure 2e), rather ohmic response is observed in the form of an approximate straight line, as expected for a symmetric structure. If the device is then measured at $50 \mathrm{mV} \mathrm{s}^{-1}$ (low scan rates), as in Figure 1, preferential injection in the quadrant initially polarized is observed again. The effect of scan rate seems to be crucial as the increment in current is induced at low enough values (a full set of scan rate values is shown as supporting information). Importantly, the order in which the different scan rates curves are measured matters in the final recorded response. Indeed, a $j-V$ curve measured at $2000 \mathrm{mV} \mathrm{s}^{-1}$ after biasing the device at slow scan of $50 \mathrm{mV} \mathrm{s}^{-1}$ does not suppress the large injection current that equals that observed in the slow measurement, 
as below demonstrated. In other words, this fact moves us to consider that modification of the injection properties at outer interfaces needs of long time poling (very slow kinetics) to be effective, as the device shows some memory on the previous history/measurements.
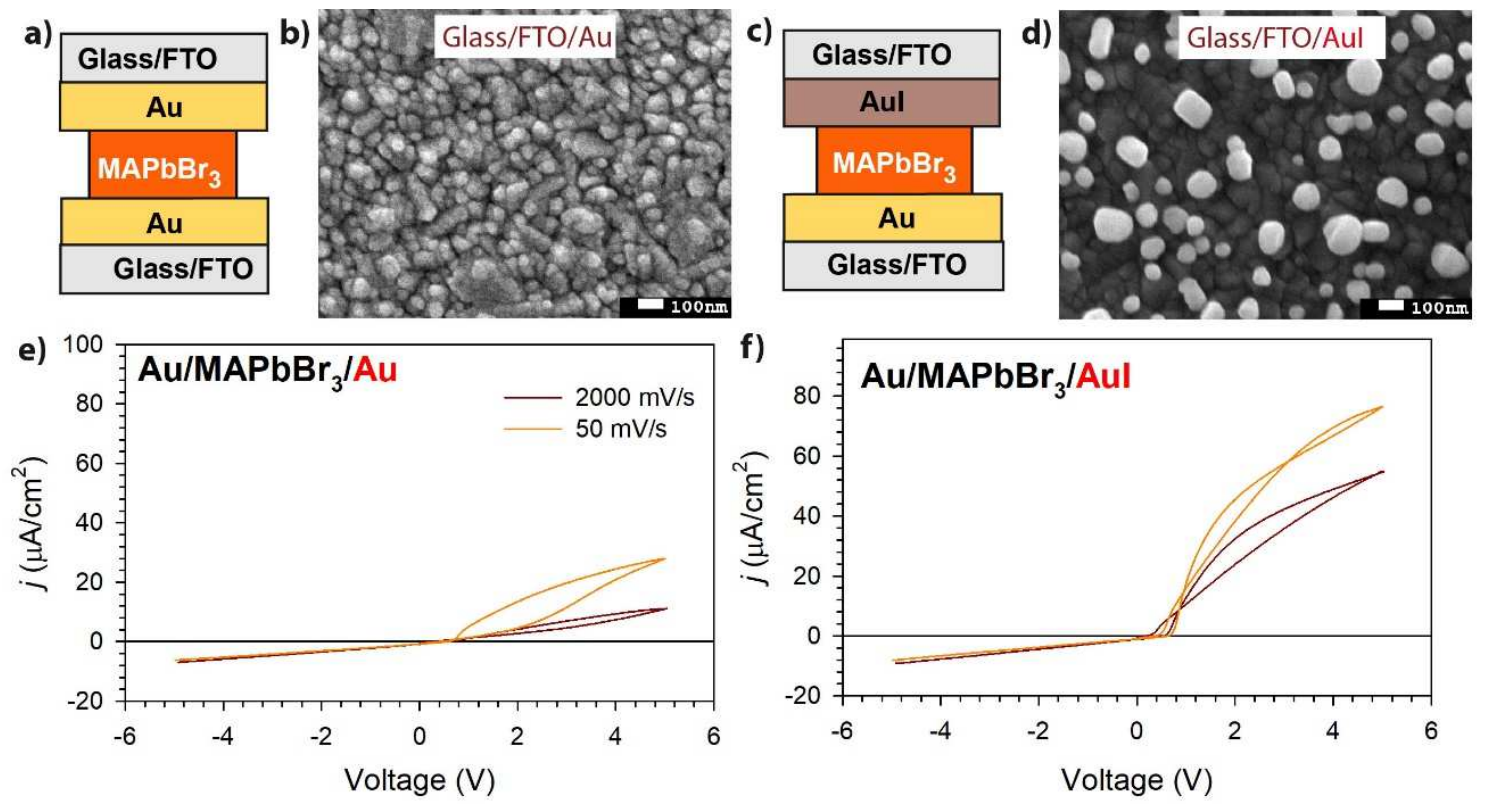

Figure 2: a) and c) Sandwiched device configuration used to record the $j-V$ curves shown in e) and f) from fresh devices, devices measured first at 2000 $\mathrm{mV} / \mathrm{s}$ followed by $50 \mathrm{mV} / \mathrm{s}$. Top view SEM images of b) Glass/FTO/Au and d) Glass/FTO/Au-I electrodes.

One candidate process accounting for the interface reactivity is the generation of species containing Au-halogen bonds fostered by intrinsic ion migration upon biasing. In order to assess this mechanism, Au-halogen contact electrodes are pre-synthesized in the configuration Glass/FTO/Au-I, and their electrical response is compared with a Glass/FTO/Au reference electrode (Figure 2a and 2c). Here, it is important to note that the chemistry of $\mathrm{Au}$ with the different halogens $(\mathrm{Cl}, \mathrm{Br}$ and $\mathrm{I})$ is very similar, as reported elsewhere. ${ }^{[23,24]}$ The Au-halogen contact electrode is synthesized by sublimation of molecular iodine onto a thin layer of evaporated gold. As can be observed in the SEM images by comparison to the reference Glass/FTO/Au (Figure 3a), formation of Au-I (Figure $3 b$ ) does not take place homogeneously leaving unreacted $\mathrm{Au}$ islands (bright spots). EDX confirms the presence of iodine atoms in the films. Alternatively, Raman spectroscopy (Figure 3a) reveals three bands in the region 100-195 $\mathrm{cm}^{-1}$, wavenumbers typical for Au-I (III) bonds with plasmon resonance signal for gold particles beyond 250 $\mathrm{cm}^{-1} .{ }^{[25]}$ The following reaction is then proposed for modified electrodes,

$$
2 \mathrm{Au}(0)+3 \mathrm{I}_{2} \text { (vapor) } \rightarrow 2 \mathrm{AuI}_{3}(\mathrm{III})
$$


where gold is oxidized and iodine is reduced. However, the reaction can be much more complex as $\mathrm{Au}$ chemistry is very rich and up to three different oxidation states have been reported to co-exist in some gold halides. ${ }^{[23]}$

Then, modified FTO/Au-I electrodes were used as external contacts in asymmetric configuration by sandwiching the perovskite monocrystal with a reference electrode of Glass/FTO/Au (Figure 2c). $j$ - $V$ curves were measured for the asymmetric configuration as shown in Figure $2 \mathrm{f}$ where high injection of carriers occurs in the pre-reacted electrode, regardless of the scan rate used during the measurement. Moreover, although the pre-reacted electrode exhibits larger current levels, the effect of favoring current injection is observed to be highly similar (Figure 2e and Figure 2f) irrespective of the halogen source, either extrinsic by iodine vapor sublimation of $\mathrm{Au}$ electrodes, or intrinsic by bias-driven migration of bromide ions as next shown.

Considering the importance of the change in the chemical environment of the external contacts due to the migrating halide ions, here we propose that gold contact reacts with mobile bromide defects to form Au-Br bonds (Figure 4). Very importantly, this chemical reactivity takes place in the same time scale (seconds-minutes) as the sweep in voltages during the $j$ - $V$ curve measurement. Here we polarize devices at $+5 \mathrm{~V}$, (see below Figure 5), and observe that if devices are pre-poled, high injection currents are observed at positive voltages even when the scan rate is $2000 \mathrm{mV} \mathrm{s}^{-1}$. This electrical response readily entails that the pre-poled process modifies the contact properties. Interestingly, a close inspection by Raman spectroscopy (Figure 3a) of an external electrode of Glass/FTO/Au that has been previously incorporated in a symmetric device and poled shows bands in the region $80-200 \mathrm{~cm}^{-1}$ that are not present in the pristine reference FTO/Au electrode. This is a strong evidence that there are new species comprising oxidized Au such as Au-X (I) or Au-X (III) bonds (X = I, Br) being formed at the surface of the electrodes, either by effect of sample biasing or vapor sublimation. Further characterization was carried out by XPS as shown in Figure $3 b$ by registering a non-polarized Au sample (Glass/FTO/Au) and a polarized sample (Glass/FTO/AuI). The binding energy $(\mathrm{BE})$ at the maximum of the $\mathrm{Au} 4 \mathrm{f}_{7 / 2}$ and $4 \mathrm{f}_{5 / 2}$ signals was found around 84.0 and $87.7 \mathrm{eV}$, respectively, which means a separation for the spin-orbit components about $3.7 \mathrm{eV}$. Further details on the analysis is provided as supporting information. These values are typical for pure metallic $\mathrm{Au}{ }^{[26,27]}$. But also, a slight difference is observed in the signal obtained of Au polarized, an asymmetric widening of both contributions of $\mathrm{Au} 4 \mathrm{f}$ signal was observed towards higher BE, as is shown in Figure $3 \mathrm{~b}$. This asymmetric widening can be assigned to the presence of oxidized species of $\mathrm{Au}$, which can be generated due to Au-halogen bonds. Typically, these Au species show a signal slightly shifted towards higher BE. ${ }^{[27,28]}$ The reversible nature of the chemical reaction is probed by repeating Raman and XPS measurements after 6 months, see supporting information. After this period of time, only metallic gold is detected using both techniques. Our findings indicate that electrode reactivity and enhancement of injection currents are closely related phenomena. 


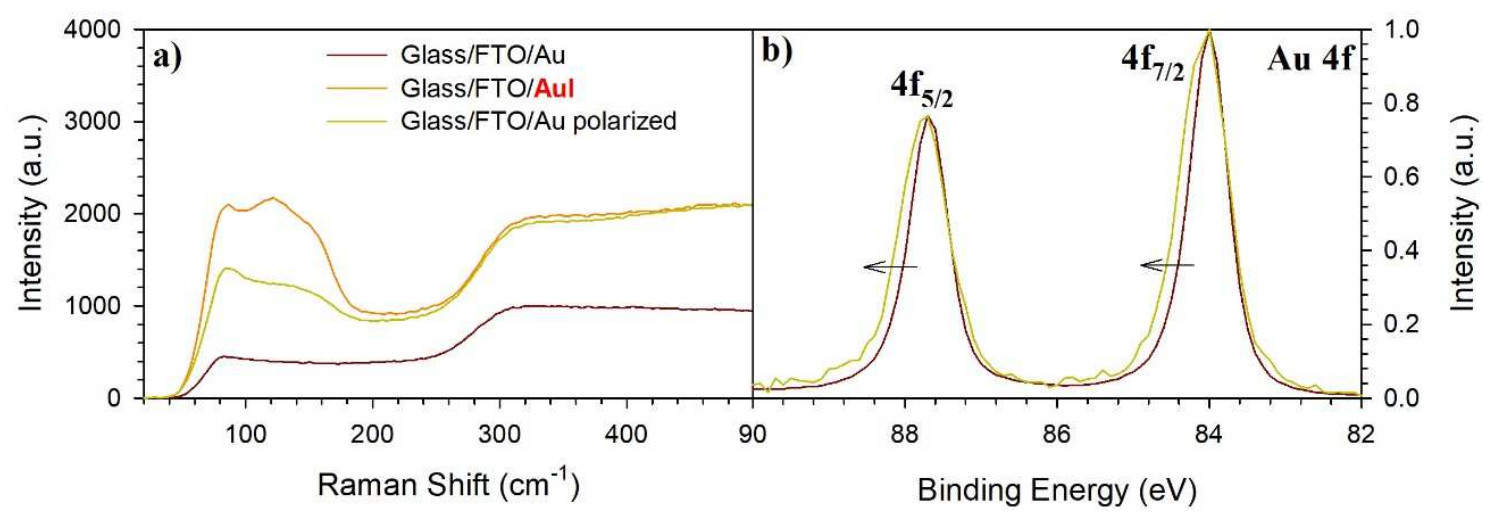

Figure 3: a) Raman spectra of Glass/FTO with different coatings containing fresh gold, pre-synthesized AuI and a gold contact that has been polarized making part of a symmetric device. b) Similar for XPS measurements indicating (arrow) the asymmetrical band widening.

We note here that the basic coordination and redox chemistry using different halides should be similar. See for example, early work by Braunstein el al. where synthesis of $\mathrm{Au}(\mathrm{I})$ and $\mathrm{Au}(\mathrm{III})$ is carried using similar chemistry for the three halides. ${ }^{[29]}$ Of course, the reaction of the halide with the metal will take place with the halide according to the standard electrode potential of each of the halogens following the series $\mathrm{Cl}>\mathrm{Br}>\mathrm{I}$ being the most favored the reaction with $\mathrm{Cl}_{2} \cdot{ }^{[30]}$ However, the nature of the species generated by the different halides would depend on the halide used. i.e. $\mathrm{Au}$ (I) vs $\mathrm{Au}$ (III). Similarly, careful consideration on the thermodynamics and kinetics of this redox chemistry will be important when selecting the electrode metal as it will determine if this type of reaction will take place. We expect metals with negative standard electrode potentials (i.e. $\mathrm{Ca}, \mathrm{Na}$ or $\mathrm{Al}$ ) to readily react with the perovskite leading to irreversible reactions. Alternatively, metal with positive standard electrode potentials such like Ag, $\mathrm{Pt}$ or $\mathrm{Au}$ should better stand the highly oxidative conditions, in some cases leading to reversible reactions. In any case, each combination metal electrode/perovskite should be carefully chosen.

Formation of oxidized $\mathrm{Au}$ (I) or $\mathrm{Au}(\mathrm{III})$ electrode is explained relying on the diagram shown in Figure 4. When an external bias is applied, the positive contact is charged with a large concentration of holes, which intervene in the metal contact oxidation (Fig 4a). The fact that halide ions are spatially close to $\mathrm{Au}$ atoms, and that $\mathrm{Pb}-\mathrm{X}$ bonds are relatively weak, halide exchange between metallic centers can be stabilized by forming $\mathrm{Au}(\mathrm{I})$ or $\mathrm{Au}$ (III) (Fig 4b). On the perovskite side of the interface, this halogen exchange leads to formation of a halide vacancy $\left(\mathrm{V}_{\mathrm{X}}{ }^{+}\right)$, as those recently reported during observation of gold interdigitated electrodes. ${ }^{[31]}$ In relation to this fact, we observed the signature of intrinsic ionic migration by using impedance spectroscopy for monocrystals of $\mathrm{MAPbBr}_{3}{ }^{[32]}$ In the long term upon continuous biasing, halide migration through a vacancy mechanism occur towards the negative electrode, that would lead to increased concentration of iodine ions in the positive electrode as observed by XPS measurements 
in a different study. ${ }^{[33]}$ 

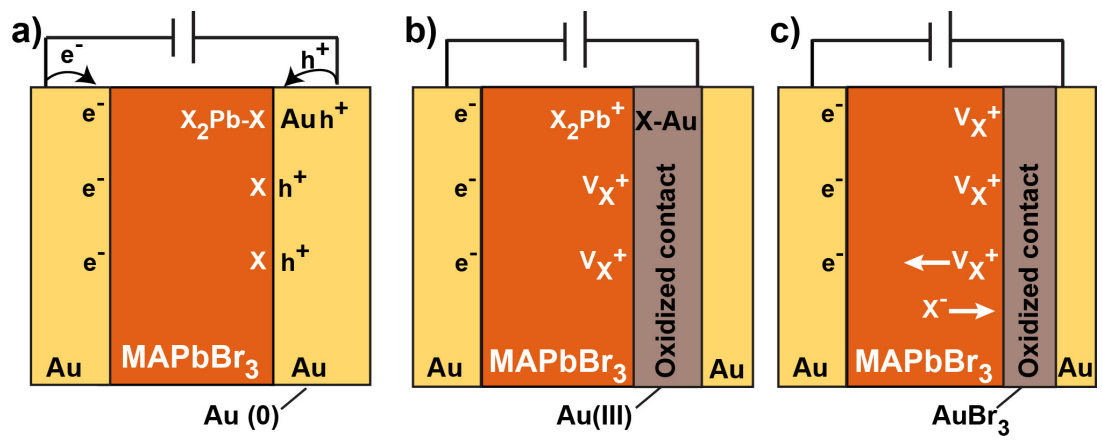

Figure 4: Proposed reaction mechanism and relationship with $\mathrm{V}_{\mathrm{X}}{ }^{+}$formation. a) Positively polarized electrode accumulates holes that gives rise to the oxidation of $\mathrm{Au}, \mathrm{b}$ ) with the formation of metal halide compounds and halide vacancies $\mathrm{V}_{\mathrm{X}}{ }^{+}$. c) This mechanism is not restricted to contacting layers but favored by the vacancy migration to the perovskite bulk.

As noted above, the halogen exchange leads to the formation of halide vacancies $\left(\mathrm{V}_{\mathrm{X}}{ }^{+}\right)$ in the perovskite side of the interface. This might lead one to consider the current increment observed upon biasing as exclusively originated by the transport of vacancies from the positively polarized electrode to the interior of the perovskite bulk (Fig. 4c). However, the current has dominant electronic component as next explained. In a hypothetical ionic mechanism as a sort of electrochemical current generation, a continuous oxidation of the gold sustains the charge flux until the total consumption of one of the reactants, either $\mathrm{Au}^{+}$or $\mathrm{X}^{-}$, leads to a current drop. By examining Fig. 5a using a fresh sample, one can infer that long time biasing $(+5 \mathrm{~V})$ produces an almost constant current after 8 hours and, therefore one can safely discard this possibility. By integration of the chronoamperometric profile, we calculate a total charge approximately equal to $Q \sim 0.2 \mathrm{C} \mathrm{cm}^{-2}$ intervening in the experiment. In order to keep the current flowing by Au electrode oxidation, the thickness of the metallic contact $d=Q v / e$ should be as large as $850 \mathrm{~nm}$, being $e$ the elementary charge. This last calculation assumes a gold unit cell volume of $v=0.0679 \mathrm{~nm}^{3}$. However, the actual gold contact is only 60 nm-thick. It is should be also stressed the incompatibility between the current response of Fig. 5a, exhibiting a steady increase over time, and models that predict current decays caused by ion-blocking, non-reacting electrodes. ${ }^{[34]}$

One can estimate the hypothetical density of formed halide vacancies after $15 \mathrm{~h}$ of continuous polarization considering the thickness of the perovskite layer $\sim 1 \mathrm{~mm}$. The $\mathrm{V}_{\mathrm{X}}{ }^{+}$density results as high as $10^{19} \mathrm{~cm}^{-3}$, certainly a physically unrealistic value that would imply a huge defect density alteration, as the sample is a monocrystal with typical reported densities of about $10^{12} \mathrm{~cm}^{-3}$. ${ }^{[35]}$ The experiment in Fig. 5b corroborates the electronic origin for the current mechanism. In the case of being the current exclusively of ionic origin, a significant current reduction is expected for high-rate biasing because ionic transport-limited mechanisms. On the contrary, large current levels are reported, 
even for high scan rates (2000 $\left.\mathrm{mV} \mathrm{s}^{-1}\right)$, as noted also previously. Therefore, our findings indicate that the reactivity of the contact, by oxidation of the gold electrode and the formation of metallic halide at the interface, is able to modify the injection properties for electronic carriers that ultimately are the responsible for the measured current.
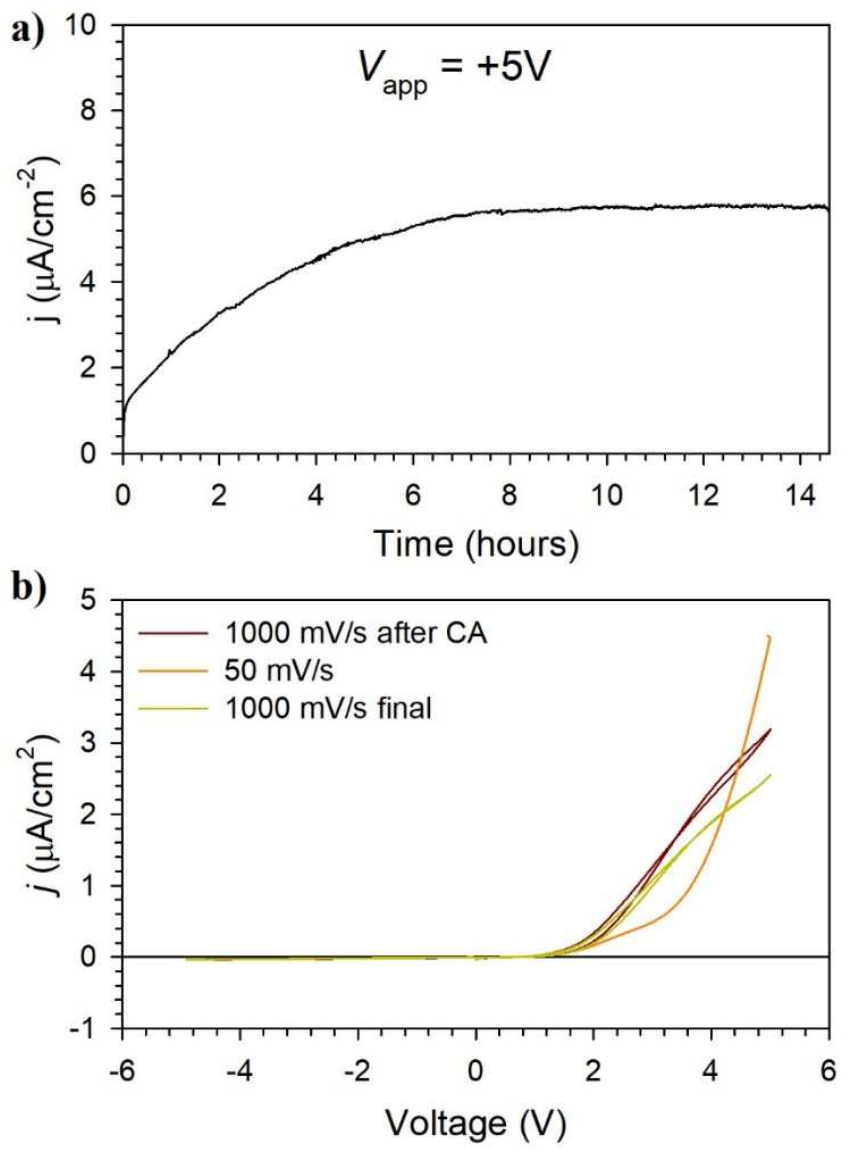

Figure 5: a) Long-time chronoamperometric experiment $(15$ h) using symmetrical $\mathrm{Au} / \mathrm{MAPbBr}_{3} / \mathrm{Au}$ devices. Current reaches rather constant levels after hours of continuous polarization. b) Sequence of biasing experiment after chronoamperometric polarization following fast-slow-fast scan rates to corroborate the electronic origin of the current increment at positive bias.

\section{Conclusions}

In this work, we have investigated the importance of the chemical reactivity of $\mathrm{Au}$ electrode in contact with single-crystal perovskite semiconductor layers. This contact has been the preferred choice in several electronic applications. It is proposed that specific interface reactivity occurs through the generation of species containing $\mathrm{Au}$ halogen bonds fostered by intrinsic ion migration upon biasing. The consequence of having metal halide compounds at the electrode interface is the net increase of the electrical current. The effect of favoring current injection is observed to be highly 
similar irrespective of the halogen source, either extrinsic by iodine vapor sublimation of $\mathrm{Au}$ electrodes, or intrinsic by bias-driven migration of bromide ions. The overall current mechanism can be rationalized as a modification of the injection properties (lowering potential barriers) of electronic carriers at the outer interface by intrinsic mobile ion electrode reactivity originating dipole-like interlayers. Contact reactivity then dominates the operation characteristics, rather than being governed by bulk transport properties of the charge carriers, either electronic or ionic. In addition, it is observed that the $\mathrm{Au}$ electrode oxidation is a highly reversible process that follows rather slow kinetics.

\section{Experimental Section}

The $\mathrm{MAPbBr}_{3}$ single crystal with variable dimensions, typically in around $3 \times 3 \times 1 \mathrm{~mm}^{3}$, was prepared by an inverse temperature crystallization technique. ${ }^{[21]}$ Lead bromide $\left(\mathrm{PbBr}_{2}, \mathrm{TCI}\right)$ and methylammonium bromide $\left(\mathrm{CH}_{3} \mathrm{NH}_{2} \cdot \mathrm{HBr}, \mathrm{MABr}\right.$, Dyesol $)$ were dissolved in $\mathrm{N}, \mathrm{N}$-dimethylformamide (DMF, Aldrich) to obtain a $1 \mathrm{M}$ solution of $\mathrm{MAPbBr}_{3}$. This solution was then filtered with a PVDF filter $(0.45 \mu \mathrm{m})$ into a clean vial. The synthesis of $\mathrm{MAPbBr}_{3}$ crystals took place in an oil bath. The initial temperature was set at $60^{\circ} \mathrm{C}$. The solution was kept at this temperature for $30 \mathrm{~min}$, and then the temperature was increased every 15 minutes by $5^{\circ} \mathrm{C}$. Finally, to grow the $\mathrm{MAPbBr}_{3}$ crystal of required size, the solution was kept for $30 \mathrm{~min}$ at $80^{\circ} \mathrm{C}$. The crystal was then extracted from the solution, rinsed in diethylether and dried with a nitrogen stream.

Devices were prepared by evaporation of gold $(60 \mathrm{~nm})$ onto two opposite sides of the crystals with an active area of $4 \mathrm{~mm}^{2} . j-V$ and polarization curves of the $\mathrm{MAPbBr}_{3}$ crystal was carried out using an Autolab PGSTAT-30. First, $j$ - $V$ curves were measured from non-previously measured crystals from zero voltage without any polarization. Then we polarized the crystal with positive $(+5 \mathrm{~V})$ or negative voltage $(-5 \mathrm{~V})$ and immediately measured $j$ - $V$ curves, again, from zero voltage. We also investigated the effect of measuring $j-V$ curves from $0 \mathrm{~V}$ towards negative values and poling during $120 \mathrm{~s}$ at either $+5 \mathrm{~V}$ or $-5 \mathrm{~V}$.

Electrodes containing Glass/Au/Au-I were prepared by evaporation of $60 \mathrm{~nm}$ of gold on glass/FTO substrate and then promoting the chemical reaction by placing upside down the electrode in the lid of a petri dish and sublimation of iodine crystals from the bottom of the Petri dish during 12 hours. In this way we obtained AuI/AuI 3 with islands of unreacted gold. Devices were prepared by sandwiching a $\mathrm{MAPbBr}_{3}$ crystal with a soft separator between Glass/FTO/Au electrode and a second electrode that can be either Glass/FTO/Au or Glass/FTO/Au-I. $j$ - $V$ curves of two different structures were measured $\mathrm{Au}-\mathrm{MAPbBr}_{3}-\mathrm{Au}$ and $\mathrm{Au}-\mathrm{MAPbBr} 3-\mathrm{Au}-\mathrm{I}$.

Raman spectroscopy measurements were carried out with a dispersive spectrometer NRS-3100 (Jasco) with energies of $80 \mathrm{~mW}$ and excitation times of $1 \mathrm{~s}$ used with a holographic filter with a cutoff edge at $70 \mathrm{~cm}^{-1}$. X-ray photoelectron spectroscopy 
(XPS) was used to study the Au chemical bonding states in the samples. The spectra were registered by using a Kratos® Axis UltraDLD spectrometer, with monochromated $\mathrm{Al}-\mathrm{K} \alpha$ radiation $(1486.6 \mathrm{eV})$ and a $20 \mathrm{eV}$ pass energy. Electrostatic charging effects could be stabilized with the help of a specific device developed by Kratos®.

\section{Acknowledgements}

This work was supported by the Czech Science Foundation under grant No. 19-23718S, and by MINECO of Spain under project MAT2016-76892-C3-1-R.

\section{TOC}

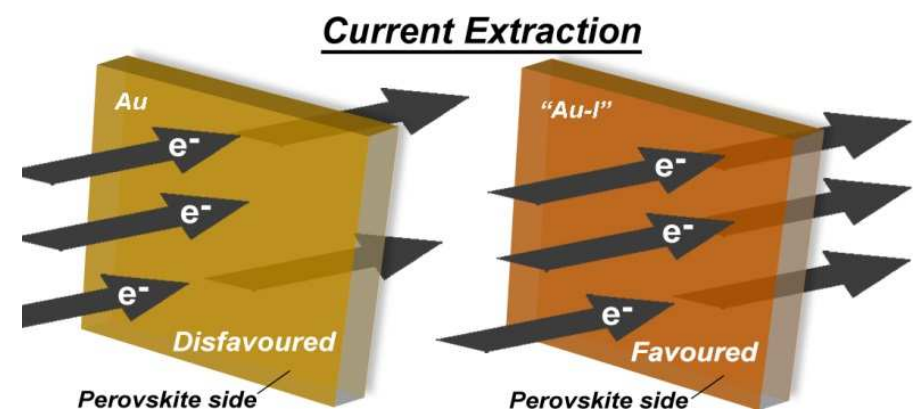

\section{References}

[1] M. A. Green, Y. Hishikawa, E. D. Dunlop, D. H. Levi, J. Hohl-Ebinger, A. W. Y. Ho-Baillie, Prog. Photovoltaics Res. Appl. 2017, 26, 3.

[2] W. Heiss, C. Brabec, Nat. Photonics 2016, 10, 288.

[3] S. Yakunin, M. Sytnyk, D. Kriegner, S. Shrestha, M. Richter, G. J. Matt, H. Azimi, C. J. Brabec, J. Stangl, M. V. Kovalenko, W. Heiss, Nat. Photonics 2015, 9, 444.

[4] H. Wei, Y. Fang, P. Mulligan, W. Chuirazzi, H.-H. Fang, C. Wang, B. R. Ecker, Y. Gao, M. A. Loi, L. Cao, J. Huang, Nat. Photonics 2016, 10, 333.

[5] S. Shrestha, R. Fischer, G. J. Matt, P. Feldner, T. Michel, A. Osvet, I. Levchuk, B. Merle, S. Golkar, H. Chen, S. F. Tedde, O. Schmidt, R. Hock, M. Rührig, M. Göken, W. Heiss, G. Anton, C. J. Brabec, Nat. Photonics 2017, 11, 436.

[6] X. Wang, D. Zhao, Y. Qiu, Y. Huang, Y. Wu, G. Li, Q. Huang, Q. Khan, A. Nathan, W. Lei, J. Chen, Phys. Status Solidi RRL 2018, 12, 1800380.

[7] O. Almora, I. Zarazua, E. Mas-Marza, I. Mora-Sero, J. Bisquert, G. GarciaBelmonte, J. Phys. Chem. Lett. 2015, 6, 1645.

[8] O. Almora, A. Guerrero, G. Garcia-Belmonte, Appl. Phys. Lett. 2016, 108, 043903.

[9] S. Yakunin, D. N. Dirin, Y. Shynkarenko, V. Morad, I. Cherniukh, O. 
Nazarenko, D. Kreil, T. Nauser, M. V. Kovalenko, Nat. Photonics 2016, 10, 585.

[10]H. Wei, D. DeSantis, W. Wei, Y. Deng, D. Guo, T. J. Savenije, L. Cao, J. Huang, Nat. Mater. 2017, 16, 826.

[11] Y. He, L. Matei, H. J. Jung, K. M. McCall, M. Chen, C. C. Stoumpos, Z. Liu, J.

A. Peters, D. Y. Chung, B. W. Wessels, M. R. Wasielewski, V. P. Dravid, A. Burger, M.

G. Kanatzidis, Nat. Commun. 2018, 9, 1609.

[12] A. Guerrero, J. You, C. Aranda, Y. S. Kang, G. Garcia-Belmonte, H. Zhou, J. Bisquert, Y. Yang, ACS Nano 2016, 10, 218.

[13] N. Sultana, N. J. Demarais, D. Shevchenko, P. J. Derrick, Solar RRL 2018, 2, 1800022.

[14] A. Guerrero, A. Bou, G. Matt, O. Almora, T. Heumüller, G. Garcia-Belmonte, J. Bisquert, Y. Hou, C. Brabec, Adv. Energy Mater. 2018, 8, 1703376.

[15] J. Carrillo, A. Guerrero, S. Rahimnejad, O. Almora, I. Zarazua, E. Mas-Marza, J. Bisquert, G. Garcia-Belmonte, Adv. Energy Mater. 2016, 6, 1502246.

[16] A. Fakharuddin, L. Schmidt-Mende, G. Garcia-Belmonte, R. Jose, I. Mora-Sero, Advanced Energy Materials 2017, 7, 1700623.

[17]H. Zhang, X. Ren, X. Chen, J. Mao, J. Cheng, Y. Zhao, Y. Liu, J. Milic, W.-J. Yin, M. Grätzel, W. C. H. Choy, Energy \& Environmental Science 2018, 11, 2253.

[18] J. A. Steele, W. Pan, C. Martin, M. Keshavarz, E. Debroye, H. Yuan, S. Banerjee, E. Fron, D. Jonckheere, C. W. Kim, W. Baekelant, G. Niu, J. Tang, J. Vanacken, M. Van der Auweraer, J. Hofkens, M. B. J. Roeffaers, Adv. Mater. 2018, 30, 1804450.

[19] K. Domanski, J.-P. Correa-Baena, N. Mine, M. K. Nazeeruddin, A. Abate, M. Saliba, W. Tress, A. Hagfeldt, M. Grätzel, ACS Nano 2016, 10, 6306.

[20] S. Cacovich, L. Ciná, F. Matteocci, G. Divitini, P. A. Midgley, A. Di Carlo, C. Ducati, Nanoscale 2017, 9, 4700.

[21] M. I. Saidaminov, A. L. Abdelhady, B. Murali, E. Alarousu, V. M. Burlakov, W. Peng, I. Dursun, L. Wang, Y. He, G. Maculan, A. Goriely, T. Wu, O. F. Mohammed, O. M. Bakr, Nat. Commun. 2015, 6, 7586.

[22] R. Gottesman, P. Lopez-Varo, L. Gouda, J. A. Jimenez-Tejada, J. Hu, S. Tirosh, A. Zaban, J. Bisquert, Chem 2016, 1, 776.

[23]D. Schröder, R. Brown, P. Schwerdtfeger, X.-B. Wang, X. Yang, L.-S. Wang, H. Schwarz, Angew. Chem. Int. Ed. 2003, 42, 311.

[24] M. Baya, A. Pérez-Bitrián, S. Martínez-Salvador, A. Martín, J. M. Casas, B. Menjón, J. Orduna, Chemistry - A European Journal 2017, 24, 1514.

[25] G. Socrates, Infrared and Raman Characteristic Group Frequencies. Tables and Charts, John Wiley \& Sons Ltd., 2001. 
[26] M. P. Casaletto, A. Longo, A. Martorana, A. Prestianni, A. M. Venezia, Surf Interface Anal 2006, 38, 215.

[27]A. K.-V. A. V. G. Naumkin, S. W.; C. J. Powell, in NIST Standard Reference Database 20, Version 4.1, Gaithersburg 2012.

[28]H. Kitagawa, N. Kojima, T. Nakajima, J Chem Soc Dalton 1991, 3121.

[29]P. Braunstein, R. J. H. Clark, Journal of the Chemical Society, Dalton Transactions 1973, 1845.

[30]D. R. L. T. J. B. T. F. Group, CRC Handbook of Chemistry and Physics, 95th Edition, CRC Press, 2014: CRC Handbook of Chemistry and Physics, 2014.

[31] C. Li, A. Guerrero, S. Huettner, J. Bisquert, Nat. Commun. 2018, 9, 5113.

[32] W. Peng, C. Aranda, O. M. Bakr, G. Garcia-Belmonte, J. Bisquert, A. Guerrero, ACS Energy Lett. 2018, 3, 1477.

[33] C. Li, S. Tscheuschner, F. Paulus, P. E. Hopkinson, J. Kießling, A. Köhler, Y. Vaynzof, S. Huettner, Adv. Mater. 2016, 28, 2446.

[34] T.-Y. Yang, G. Gregori, N. Pellet, M. Grätzel, J. Maier, Angew. Chem. Int. Ed. 2015, 54, 7905

[35] W. Peng, L. Wang, B. Murali, K.-T. Ho, A. Bera, N. Cho, C.-F. Kang, V. M. Burlakov, J. Pan, L. Sinatra, C. Ma, W. Xu, D. Shi, E. Alarousu, A. Goriely, J.-H. He, O. F. Mohammed, T. Wu, O. M. Bakr, Adv. Mater. 2016, 28, 3383. 


\section{Supplementary Information}

\section{Reversible Formation of Gold Halides in Single-Crystal Hybrid-Perovskite/Au Interface upon Biasing and Effect on Electronic Carrier Injection}

Jan Pospisil ${ }^{1}$, Antonio Guerrero ${ }^{2 *}$, Oldrich Zmeskal $^{1}$, Martin Weiter ${ }^{1}$, Juan Jesus Gallardo, ${ }^{3}$ Javier Navas, ${ }^{3}$ and Germà Garcia-Belmonte ${ }^{2 *}$

${ }^{1}$ Brno University of Technology, Faculty of Chemistry, Purkyňova 118, 61200 Brno, Czech Republic

${ }^{2}$ Institute of Advanced Materials (INAM), Universitat Jaume I, 12006 Castelló, Spain

${ }^{3}$ Departamento de Química Física, Facultad de Ciencias, Universidad de Cádiz, E11510 Puerto Real (Cádiz), Spain

Email: aguerrer@uji.es, garciag@uji.es

27 August 2019 


\section{J-V curves}

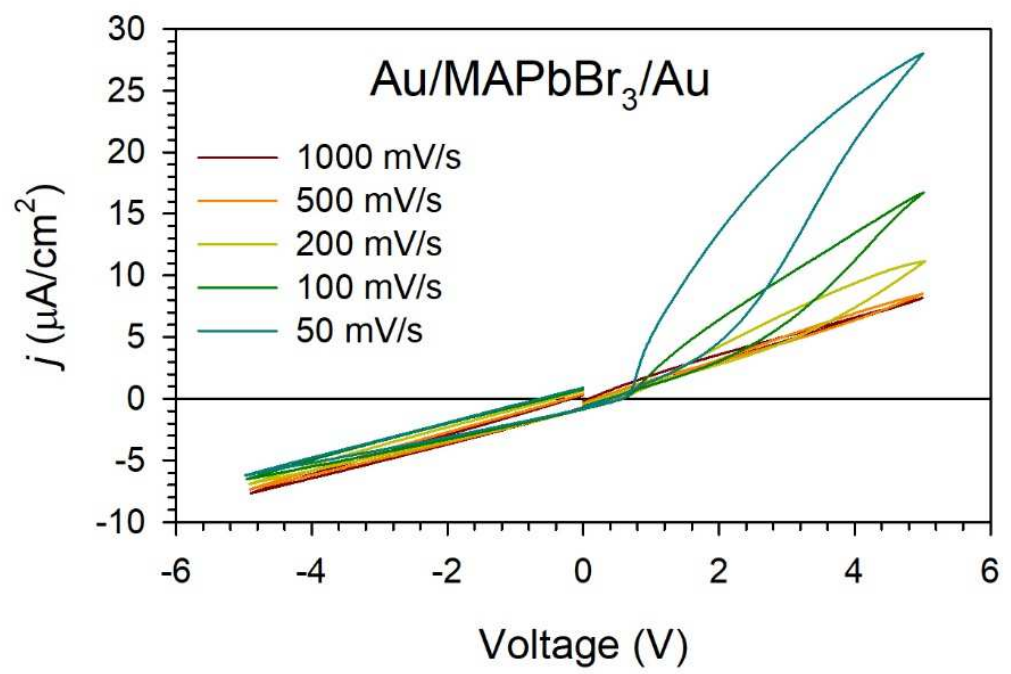

Figure $\mathrm{S} 1: j-V$ curves of a fresh device in the configuration $\mathrm{Au} / \mathrm{MAPbBr} 3 / \mathrm{Au}$ measured at different scan rates starting from $1000 \mathrm{mV} / \mathrm{s}$, initially from $0 \mathrm{~V}$ towards positive voltages.

\section{Raman and XPS Analysis}

In order to analyse the chemical state of the Au layer in the device, Raman spectroscopy and X-ray photoelectron spectroscopy (XPS) measurements were performed. The Raman spectra were registered in a backscattering geometry using a Jobin Yvon U1000 double monochromator equipped with a Hamamatsu R-943 photomultiplier, and using a DPSS $532 \mathrm{~nm}$ laser as the illumination source. Figure S2 shows Raman spectra obtained for a non-polarized reference Au sample (coloured in black in the figure), for an Au sample polarized 6 months before (named as 6 months $\mathrm{Au}$ polarized sample and coloured in blue in the figure) and a recently Au polarized sample (coloured in red). Both, the non-polarized sample and the sample polarized several months ago show similar Raman spectra, which means both samples are quite similar. This can be an evidence of the reversible reaction occurring in the device. Also, the Raman spectrum for the recently $\mathrm{Au}$ polarized sample is different and shows the presence of a band between $80-200 \mathrm{~cm}^{-1}$, which is an evidence of $\mathrm{Au}-\mathrm{X}$ bonds $(\mathrm{X}=\mathrm{I}$, $\mathrm{Br})$ in the sample. 


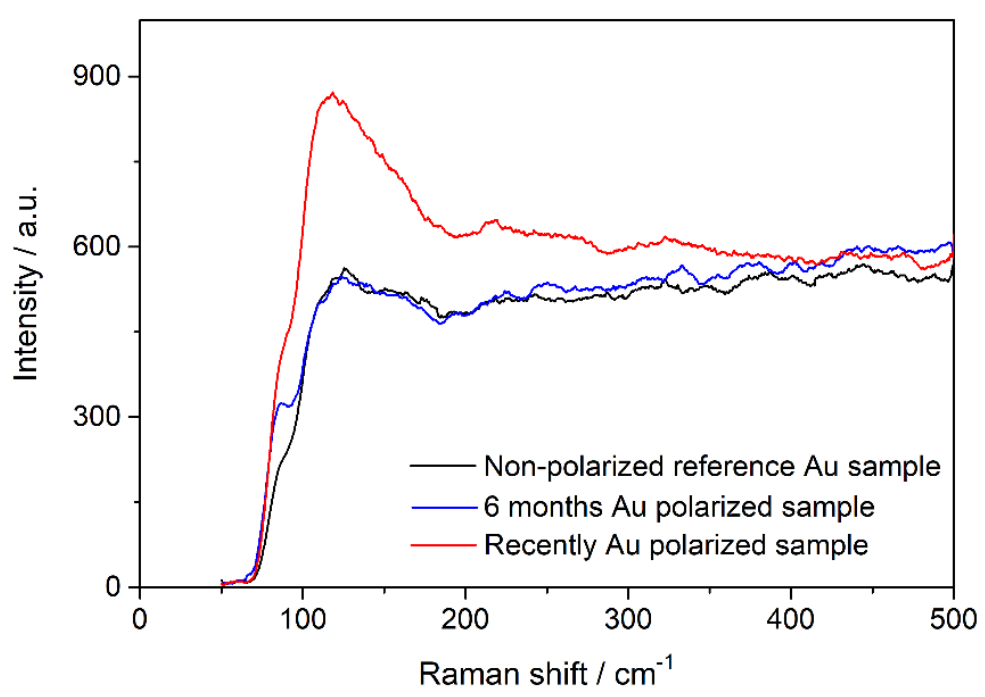

Figure S2. Raman spectra registered for a non-polarized Au sample, an Au sample polarized 6 months, and a recently Au polarized sample.

X-ray photoelectron spectroscopy was used for confirming these results and for analysing the chemical state bonding of Au. The spectra were registered by using a Kratos ${ }^{\circledR}$ Axis UltraDLD spectrometer, with monochromated Al-K $\alpha$ radiation (1486.6 $\mathrm{eV}$ ) and a $20 \mathrm{eV}$ pass energy. Electrostatic charging effects could be stabilized with the help of a specific device developed by Kratos ${ }^{\circledR}$. In some cases, the XPS measurements can produce the reduction of oxidized samples, and for this reason to avoid the reduction of possible oxidized species of Au, the XPS spectra were registered using a low level of $\mathrm{X}$-ray irradiation. Thus, Figure S3a shows the $\mathrm{Au} 4 \mathrm{f}$ spectra registered of the nonpolarized reference Au sample, for an Au sample polarized 6 months ago and a recently Au polarized sample, and Figure $\mathrm{S} 3 \mathrm{~b}$ shows the detail of the $\mathrm{Au} 4 \mathrm{f}_{7 / 2}$ contribution. For the recently polarized sample, several spectra were registered in different zones and four of them are shown in the figure (named as Au polarized). In all cases, the binding energy $(\mathrm{BE})$ at the maximum of the $\mathrm{Au} 4 \mathrm{f}_{7 / 2}$ and $4 \mathrm{f}_{5 / 2}$ signals was around 84.0 and 87.7 $\mathrm{eV}$, respectively, which means a separation for the spin-orbit components about $3.7 \mathrm{eV}$. These values are typical for pure metallic $\mathrm{Au} \stackrel{1}{1} \underline{2}$. As is observed in the Figure, the nonpolarized and the polarized 6 months ago samples show quite similar Au 4f signals, which means $\mathrm{Au}$ in the samples is very similar. This is coherent with the results obtained by Raman spectroscopy, which is an evidence of the reversible reaction 
occurring in the device. But, a slight difference is observed in the signal obtained for all the spectra registered of recently Au polarized sample, that is an asymmetric widening of both contributions of $\mathrm{Au} 4 \mathrm{f}$ signal was observed towards higher $\mathrm{BE}$, as is shown in Figure S3a, and for $\mathrm{Au} 4 \mathrm{f} 7 / 2$ signal, this is observed in detail in Figure S3b. This asymmetric widening can be assigned to the presence of oxidized species of $\mathrm{Au}$, which can be generated due to Au-halogen bonds. Typically, these Au species show a signal slightly shifted towards higher $\mathrm{BE} \stackrel{2}{2}, \underline{3}$.
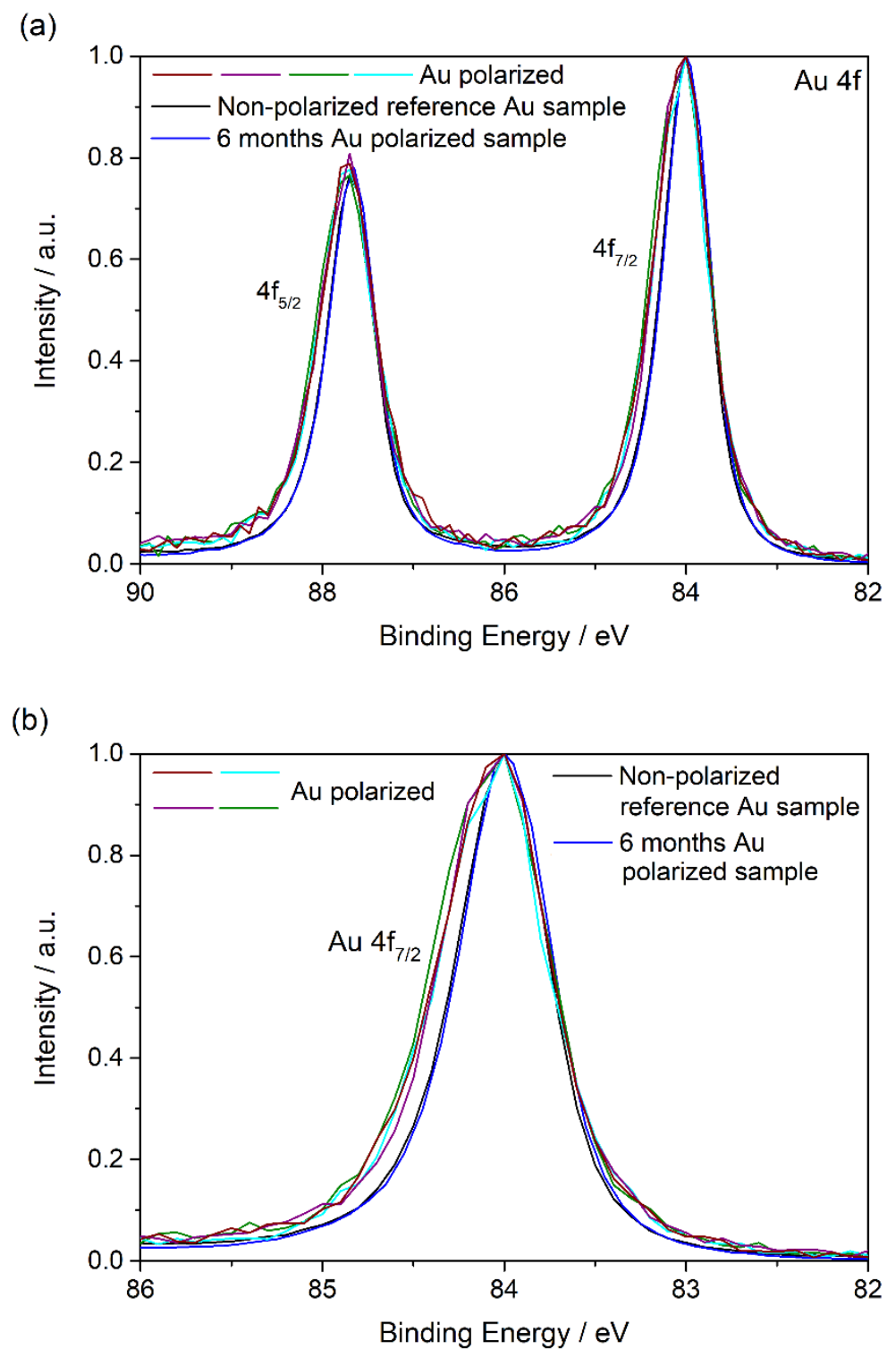

Figure S3. XPS signal for $\mathrm{Au} 4 \mathrm{f}$ (a) and in detail for $\mathrm{Au} 4 \mathrm{f}_{7 / 2}$ (b). Spectra named as $\mathrm{Au}$ polarized correspond to those registered for recently $\mathrm{Au}$ polarized sample in different zones. 


\section{References}

1. Casaletto, M. P.; Longo, A.; Martorana, A.; Prestianni, A.; Venezia, A. M., XPS study of supported gold catalysts: the role of $\mathrm{Au}-0$ and $\mathrm{Au}+\mathrm{delta}$ species as active sites. Surf Interface Anal 2006, 38, 215-218.

2. Naumkin, A. K.-V. A. V. G., S. W.; C. J. Powell, in NIST Standard Reference Database 20, Version 4.1, Gaithersburg 2012.

3. Kitagawa, H.; Kojima, N.; Nakajima, T., Studies of Mixed-Valence States in 3Dimensional Halogen-Bridged Gold Compounds, Cs2auiauiiix6 ( $\mathrm{X}=\mathrm{Cl}, \mathrm{Br}$ or I) .2. XRay Photoelectron Spectroscopic Study. J Chem Soc Dalton 1991, 3121-3125. 